\title{
Editoriale \\ Pedagogia dell'oggi: le sfide del presente. Percorsi di ricerca. Ricchezza e pluralità dell'indagine contemporanea
}

Michele Corsi, Massimiliano Stramaglia \& Mathieu Deflem*

La scelta editoriale della direzione di questa rivista, per tutto il 2020, è stata quella di dedicare l'intera annata alla ricerca pedagogica contemporanea. A rendicontazione della call che abbiamo lanciato nella seconda metà del 2019. Per la quale abbiamo ricevuto, come ci aspettavamo del resto, una quantità elevata di risposte scientificamente robuste, testimoniate dai numerosi articoli che abbiamo ospitato nel fascicolo precedente. E così di seguito, ma differenti, in questo numero. Tant'è che entrambi i dossier risultano di una tale mole da giustificare la corposità delle pagine che li compongono.

Una seconda osservazione.

Sotto la spinta dell'emergenza pandemica, reale, dovuta, razionale e, peraltro, anche emotiva e psicologicamente devastante, l'editoriale del fascicolo precedente lo abbiamo dedicato al maledetto "tempo sospeso" e inquietante, per più aspetti, del coronavirus. Un tempo mondiale, per giunta, articolato e frastagliato. Polemico e nondimeno politicizzato e ricco di contrasti. Che non accennano a diminuire. Basti pensare al conflitto tuttora perdurante tra gli USA, la Cina e l'Organizzazione Mondiale della Sanità. Con alcuni motivi che sono pure comprensibili. O almeno in parte.

Quasi mettendo in sordina la specificità dei contributi accolti, con riferimento sempre all'editoriale. E lasciando ai lettori la pressoché totale autonomia di riflessione, di giudizio, e, dunque, di possibili interpretazioni ermeneutiche e prospettiche.

In questo numero, invece, vogliamo portare l'attenzione sul tema complessivo di questi due dossier. Utilizzando i contributi qui pubblicati come una lente di ingrandimento, e pertanto di commento critico, di occupazione del presente e di lancio nel futuro, anche degli articoli del primo numero di questo 2020. Così da contenerli e osservarli globalmente, quelli e questi. Con uno sguardo onnicomprensivo capace di rendere testimonianza alla domanda: dove va la pedagogia attuale? Italiana e mondiale; per lo meno quella dei Paesi più colti e

\footnotetext{
* Professor of Sociology at the University of South Carolina. E-mail: m@mailbox.sc.edu.
} 
scientificamente più attrezzati. Quali sono i suoi filoni di ricerca, le loro ricadute "pratiche", la loro incidenza sui bisogni e le fatiche delle persone di questo tempo presente?

Fedeli al nostro principio epistemologico di una pedagogia "utile". Che, partendo dal passato, e quindi dalla storia della pedagogia e dell' educazione, indaghi attentamente, e senza pregiudizi di sorta, vizi ideologici o peggio, il qui e ora della contemporaneità umana, per proiettare, negli anni che verranno, la sua profezia lungimirante di bene individuale e comunitario.

Di altra pedagogia, con un ritornello che ci è caro, non sappiamo che farcene. Inutile, se non talora pure dannosa. Tanto da sottrarre spazio e visibilità alla stessa pedagogia, e confonderla, come negli scaffali delle migliori librerie, con la filosofia (per giunta, non sempre dell'educazione), quando non anche con la puericultura o con una claudicante scienza dell'alimentazione di taglio pediatrico. Dando così, per questa via, un maggiore risalto alla psicologia, pure dello sviluppo, della sociologia, dell'antropologia e delle scienze umane e sociali in genere.

Non tutta la pedagogia italiana, ahimè, è però coerente col suo statuto epistemologico.

Per rappresentare, talvolta, una sorta di ritorno, infelice e infausto, a un postpositivismo di vecchia maniera. Confezionata, com'è, quale una scienza di risulta o applicata alla Parsons: biologia applicata o vecchia filosofia rimaneggiata, scientismo di seconda o terza fila alla Durkheim o alla sequela di Piaget ecc. Che, mentre gli originali sono sempre nobili e altissimi, ancorché non condivisibili in ottica pedagogica, le copie, brutte o semi-belle che possono essere, sono sempre tali. Riedizioni e rifacimenti. Quando non anche artificiosi, mal fatti e di poco o nullo valore.

Ma vogliamo aprire il cuore alla speranza.

Come nei riguardi di questo Covid-19. O ne rimaniamo, infatti, schiacciati, ansiosi e spaventati, oppure, come nella migliore tradizione del teatro napoletano, osiamo pensare che la nottata prima o poi finirà.

Che tornerà il sereno, dopo la pioggia.

O l'alba, chiara e luminosa, terminato il buio, sovente pesto e sgradevole, delle ore precedenti.

Lasciamo comunque da parte le lamentazioni, pure quando dovute e necessarie. Per concentrarci sul meglio, sul futuribile e sull'attuabile: di nuovo, cioè, sull'utile e sul servibile o praticabile, alla Dewey, almeno della pedagogia italiana.

Tant'è che tutti i contributi qui ospitati, e di cui daremo conto, sono indagini, in breve, di ricerche a monte, più complesse, di colleghe e colleghi del nostro Paese. 
Una terza osservazione è legata all'attuale cambio di passo della pedagogia nazionale. E al suo transito, non sempre lieve e compreso, da un'indagine meno plurale e articolata a una ricchezza e a una numerosità investigativa di filoni e di piste euristiche. Particolarmente nell'ultimo trentennio. Come la mia stessa osservazione diretta può testimoniare. $\mathrm{E}$ che, forse, qualche collega più "critico" potrebbe anche definire eccessive.

Può darsi.

Ma un nuovo ordine nasce sempre da un disordine a monte.

Dal caos alla creazione.

Ben vengano, pertanto, le odierne e molteplici linee di ricerca. Che pure connettano meglio la pedagogia con gli interessi delle scienze sociali: il che può portare a una feconda fertilizzazione incrociata di temi e prospettive. Alcune delle quali si dovranno certamente raffinare nel tempo, per diventare pure più pedagogiche. E con altri indirizzi che si potranno anche "combinare" o unificare tra loro, per risultare meno di micro-nicchia e dotarsi di un maggiore respiro educativo, scientifico e prospettico.

Ma veniamo a questo numero e al cambiamento di esplorazione teorica e d'intervento pratico nell'ultimo trentennio pedagogico italiano. E non solo, territorialmente o geograficamente parlando.

Negli anni '70 la pedagogia nostrana (intendendo, per essa e con essa, la globalità dei nostri attuali quattro settori scientifico-disciplinari) aveva un centro d'interesse prevalente: la scuola. Tanto da fare dei pedagogisti gli unici, pressoché riconosciuti, esperti di questa istituzione.

Così da non vincere un concorso a cattedra quale professore ordinario di questa disciplina se non si aveva, nel proprio curricolo, almeno una monografia che affrontasse una siffatta tematica, ovvero l'educazione scolastica sotto un qualche aspetto di questo poliedrico cristallo.

E al suo fianco, in combinato disposto, o la pedagogia della famiglia, rigorosamente al singolare e di strettissima marca cattolica (pochi o ancora meno, infatti, i pedagogisti laici che se ne occupavano) oppure, maggiormente, la filosofia dell'educazione. Frequentata, quest'ultima, da entrambi gli schieramenti.

Solo marginalmente, e rariter, altri tipi di approfondimenti (parlo in specie di ciò che è oggi M-PED/01): un libro ogni tanto, e comunque scarsi, su qualche altro argomento da parte di taluni colleghi più avventurosi o, se vogliamo, fantasiosi. Per non tacciare di originalità unicamente alcuni a discapito di altri.

Una pedagogia, peraltro, spesso declinata con la storia della pedagogia. Così da passare indifferentemente da una cattedra all'altra.

A maggior ragione la didattica (attualmente M-PED/03), intesa unicamente, allora, come la teoria generale dell'insegnamento, e null'altro. Naturalmente scolastico. O l'iniziale pedagogia sperimentale. Più adusa negli atenei pontifici, 
particolarmente in quello salesiano, dove si scriveva di valutazione (la docimologia) o di orientamento. Pure qui, per lo più scolastici. Con sparute e timide aperture all'università.

Il cambio di passo lo abbiamo all'inizio del ' 90 , con la trasformazione dei Magisteri in Facoltà di Scienze della formazione, e con la modifica della tabella dei suoi Corsi di laurea. Al pari della dovuta e crescente attenzione al dialogo tra le pedagogie e le scienze dell'educazione.

Col 2000 e con la riforma Berlinguer, si assisterà, poi, a una vera e propria esplosione ermeneutica e investigativa anche in casa pedagogica nella sua interezza. Con una moltiplicazione d'indagini senza pari. Di cui il presente fascicolo offre una prima evidente dimostrazione.

Sicché ben undici sono i differenti tracciamenti rinvenibili in queste pagine. Con alcune diversificazioni pure al loro interno. Così da raddoppiarsi quasi ulteriormente.

E che non sono poi nemmeno tutti quelli cui le pedagogie del nostro Paese hanno dato vita nell'ultimo ventennio.

Con altri ancora che si stanno affacciando più di recente, come la pop education, o la pop pedagogia, o quella dei miti musicali.

Sullo sfondo nondimeno di una rinascente psicopedagogia o di una ripresa, sotto la spinta sempre più marcata e diffusa dei fenomeni di devianza, peggio: di violenza, della cosiddetta pedagogia di strada. Cara alle menti più illuminate dei maestri scrittori delle borgate romane (Pietralata) o delle periferie delle grandi città (da Milano a Torino, a Palermo).

E veniamo a questo numero. E ai suoi contributi.

$\mathrm{Ne}$ dò conto, senza un ordine ovviamente di merito o di consistenza settoriale o sub-settoriale. Ma seguendo, quale indicatore, i tempi della loro approvazione e, quindi, della conseguente pubblicazione.

Con alcuni ambiti, anzi, che figurano qui con una maggiore "presenza", di contro allo spazio editoriale che occupano talora nella più vasta bibliografia pedagogica, e italiana in specie: sono, infatti, tutti di colleghe e colleghi del nostro Paese gli articoli che appaiono in queste pagine. Ma nondimeno mondiale.

Due fascicoli, questi, che vanno pertanto colti come un unico volume: li abbiamo suddivisi solo per ragioni di spazio o di "peso". E di cui anche agli apporti dei pedagogisti stranieri nel primo fascicolo. O di cultori prossimi alle nostre discipline.

La pedagogia di genere al singolare e al plurale, con estensione alla maschilità e all'omofobia pure in prospettiva evolutiva e alla performance di danza: A. Ceciliani, G. Burgio, F. Buccini, M.L. Iavarone, F.I. Ambra, L. Aruta e A. Pontremoli. 
I rapporti tra la pedagogia e la psicoanalisi, sufficientemente frequentata da studiosi più che illustri già all'inizio del $\mathrm{XX}^{\wedge}$ secolo e qui riguardata sotto le lenti del desiderio, anche tra limite e bisogno: M. Corsi e M. Ilardo.

La pedagogia delle famiglie, l'educazione parentale e le azioni di counseling e homeschooling al riguardo: F. Paone, A. Chinazzi e M. Stramaglia.

La pedagogia del lavoro nelle sue varie declinazioni, pure quelle tecnologicamente più avanzate, dalle macchine intelligenti alle employabilities e alle soft skills, fino all'apprendimento in ambito universitario, con riferimento nondimeno al mentoring, all'apprendistato e all'occupazione giovanile: M. Costa, C. Pignalberi e S. Rizzari.

La città educante: F. d'Aniello; e il ruolo, o l'influenza, dei mass-media in generale sulla società, la scuola e le varie agenzie educative, e dunque sul sistema formativo integrato nella sua complessità: E.M. Bruni.

La pedagogia dell'inclusione, sia scolastica (a fronte anche della manifestazione o della differente fenomenologia del bullismo), che culturale e letteraria: V. Rossini, F. Pizzolorusso, M. Sannipoli e E. Miatto.

La didattica e le didattiche, con dovuta apertura alle frontiere del reflective teachers' training, del capability approach e della formazione alle competenze digitali, pure nei sistemi di comunicazione aumentativa alternativa per allievi con disabilità cognitive: A. Cunti, A. Priore, G. Cappuccio, M. Albanese, L. Maniscalco, S. Fontana e S. Polenta.

Gli studi sulla corporeità in senso lato, alla luce anche degli apporti della neuro-pedagogia e della neuro-didattica, sullo sfondo nondimeno delle scienze motorie, sino ai campi dell'affettività e della sessualità negli albi illustrati, per decostruire, quanto possibile, stereotipi e pregiudizi: S. Bellantonio e S. Fierli.

L'ambito delle politiche educative: L. Girotti.

Come quello della pedagogia sociale nelle sue differenti prospettive e fino ai lidi dell'educazione globale e di quella alla cittadinanza: R. Deluigi e L. Milani.

E da ultima, ma non ultima, la vasta area della pedagogia interculturale pure in chiave europea e nondimeno scolastica: S. Sani.

Un vasto caleidoscopio, quindi, di tagli investigativi, di prospettive di ricerca e di proposte pedagogico-educative su cui soffermarsi attentamente e criticamente nelle pagine di questo fascicolo. Che rinviano, apertamente o meno, anche ad altre piste d'indagine. E la cui bibliografia complessiva, ivi citata a sostegno delle tesi che sono portate, schiude ulteriormente a un intero mondo euristico che, tutto assieme, rappresenta la pedagogia italiana e mondiale dell'oggi, con le attuali sfide del presente.

Che quello che accadrà domani, nell'ambito delle nostre discipline, è storia, forse o sicuramente, tutta da leggere e, a monte, da scrivere. Nell'epoca del velocissimo cambiamento che ci riguarda. E persino ci sovrasta. 